\title{
Two Optimization Problems in Thermal Insulation
}

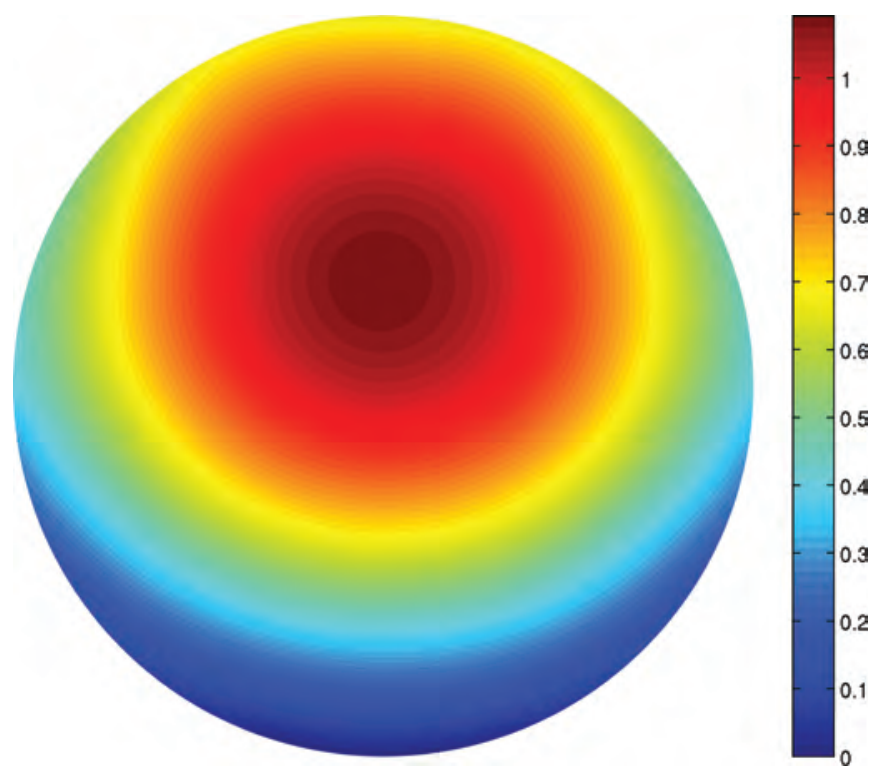

\section{Dorin Bucur, Giuseppe Buttazzo, and Carlo Nitsch}

ABSTRACT. We consider two optimization problems in thermal insulation. In both cases the goal is to find a thin layer around the boundary of the thermal body which gives the best insulation. The first problem deals with the case in which a given heat source is present. In the second case there are no heat sources, and the goal is to have the slowest decay of the temperature. In the first case, for a uniform heat source, the insulation is distributed uniformly about a ball, though for a square it is more concentrated on the middles of the sides. In the second case, even for a ball, when the amount of insulation falls below a certain threshold, it is better not to distribute it uniformly. We also provide numerical computations and a list of open questions.

Dorin Bucur is professor of mathematics at Université Savoie Mont Blanc (France). His e-mail is dorin . bucur@univ-savoie.fr. Giuseppe Buttazzo is professor of mathematics at Università di Pisa (Italy). His e-mail is gi useppe . buttazzo@uni pi . it.

Carlo Nitsch is professor of mathematics at Università di Napoli (Italy). His e-mail is car7o.nitsch@unina.it .

For permission to reprint this article, please contact:

reprint-permission@ams.org.

DOI: http://dx.doi.org/10.1090/noti1557

\section{Introduction}

Essential as they are to energy saving, pollution control, and environment improvement, insulation problems promise to be a crucial research area for the foreseeable future. Several sciences are involved: civil engineering for the design of new buildings with more efficient energy consumption, physics and chemistry for research on new materials with better insulating properties, and mathematics for the study of the partial differential equations 
for heat conduction in the presence of insulating regions.

In this short note we present two problems related to optimal insulation. In both cases a domain $\Omega$ of $\mathbb{R}^{d}$ is given. We assume it is a bounded open set with a regular boundary $\partial \Omega$. For simplicity we assume that $\Omega$ is a conducting domain with a constant conductivity coefficient, which we assume equal to one. The goal is to distribute around $\partial \Omega$ a layer $\Sigma$ of insulating material in some efficient way; the optimality criterion we use is described later. We describe the layer $\Sigma$ by means of the tangential and normal coordinates on $\partial \Omega$ :

$$
\Sigma_{\varepsilon}=\{\sigma+t \nu(\sigma): \sigma \in \partial \Omega, 0 \leq t<\varepsilon h(\sigma)\},
$$

where $v(\sigma)$ is the exterior normal versor to $\partial \Omega$ at the point $\sigma$ and the function $h$ describes the variable thickness. The index $\varepsilon$ describes the average thickness of the layer and is taken very small (perhaps a few centimeters for a small house). The conductivity coefficient $\delta$ of the insulating material in the layer $\Sigma_{\varepsilon}$ is taken very small too. Finally, we assume that the temperature is zero outside the set $\Omega \cup \Sigma_{\varepsilon}$.

The two problems we deal with are described below in precise mathematical form.

Problem 1. We put in $\Omega$ a heat source $f \in L^{2}(\Omega)$; after waiting enough time the temperature $u(t, x)$ approaches the stationary temperature $u(x)$ that is the solution of the elliptic equation with transmission conditions at $\partial \Omega$

$$
\begin{cases}-\Delta u=f & \text { in } \Omega \\ -\Delta u=0 & \text { in } \Sigma_{\varepsilon} \\ u=0 & \text { on } \partial\left(\Omega \cup \Sigma_{\varepsilon}\right) \\ \frac{\partial u^{-}}{\partial v}=\delta \frac{\partial u^{+}}{\partial v} & \text { on } \partial \Omega .\end{cases}
$$

Equivalently, the stationary temperature $u$ can be seen as the solution of the minimum problem on $H_{0}^{1}\left(\Omega \cup \Sigma_{\varepsilon}\right)$ for the energy functional

(2) $E_{\varepsilon, \delta}(u)=\frac{1}{2} \int_{\Omega}|\nabla u|^{2} d x+\frac{\delta}{2} \int_{\Sigma_{\varepsilon}}|\nabla u|^{2} d x-\int_{\Omega} f u d x$.

The PDE (1) is indeed the Euler-Lagrange equation of the minimum problem related to the cost functional (2). Denoting by $u$ the solution of the PDE (1) (or of the variational problem for the energy (2)) a multiplication by $u$ in (1) and a standard integration by parts allows us to write the minimum of the energy functional (2) in the form

$$
\min _{H_{0}^{1}\left(\Omega \cup \Sigma_{\varepsilon}\right)} E_{\varepsilon, \delta}=-\frac{1}{2} \int_{\Omega} f u d x .
$$

Note that when the heat sources are uniformly distributed the minimization of the energy functional above corresponds to the maximization of the average temperature.

The optimization problem we deal with consists in the optimal choice of the shape of the insulating layer $\Sigma_{\varepsilon}$ around $\partial \Omega$ once the total amount of insulating material is prescribed. Stressing the dependence on $h$ of the energy functional and denoting by $E(h)$ the quantity

$$
E(h)=\min _{H_{0}^{1}\left(\Omega \cup \Sigma_{\varepsilon}\right)} E_{\varepsilon, \delta},
$$

our first optimization problem seeks

$$
\min \left\{E(h): h \in \mathcal{H}_{m}\right\},
$$

where $\mathcal{H}_{m}$ denotes the class of admissible choices

$$
\mathcal{H}_{m}=\left\{h: \partial \Omega \rightarrow \mathbb{R}, h \geq 0, \int_{\partial \Omega} h d \sigma=m\right\} .
$$

This applies for instance to the thermal insulation of a house as in Figure 1 or of a pipe as in Figure 2. One of the most crucial questions in this field is: "Which parts have to be more protected?" For instance, in a radially symmetric body such as the igloo of Figure 3, should we put a layer of insulating material with a constant boundary thickness?

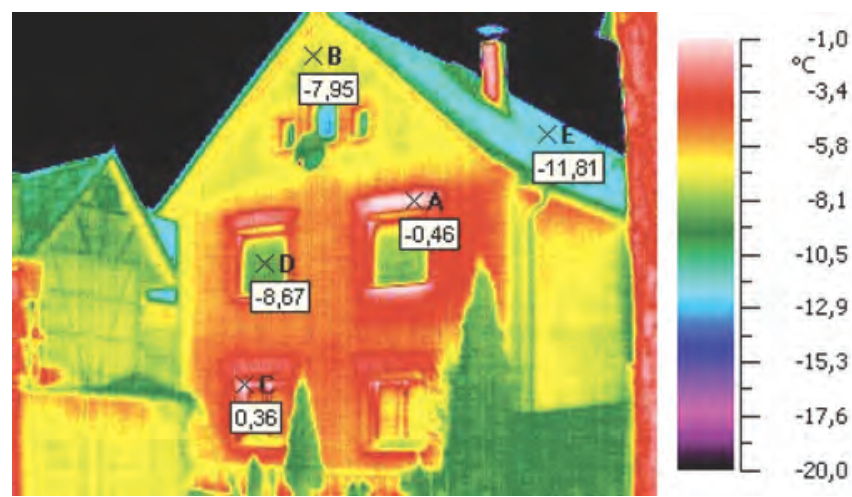

Figure 1. Heat leaves a house at different rates at different places. What is the optimal distribution of insulation?

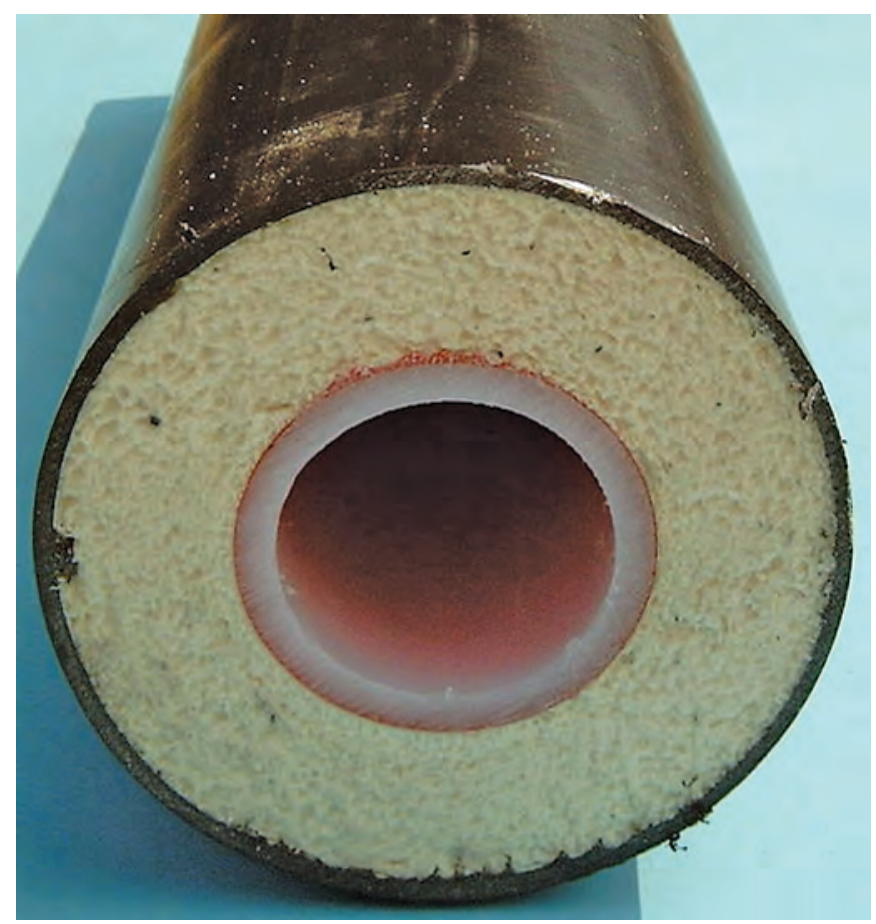

Figure 2. Pipes have substantial thermal insulation. 


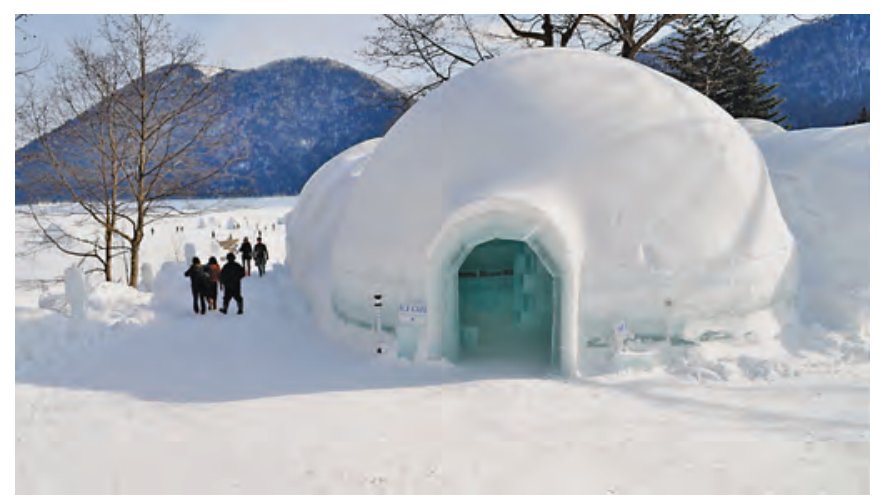

Figure 3. The thermal insulation of an igloo by a layer of snow.

Problem 2. The second problem that we consider after A. Friedman [5] deals with a domain $\Omega$ as above, with a fixed initial temperature $u_{0}$ and without any heat source. In this case the temperature decays to zero and our goal is to put the insulating material around $\Omega$ so that this decay is as low as possible. This applies for instance to the thermal insulation of a coffee pot as in Figure 4.

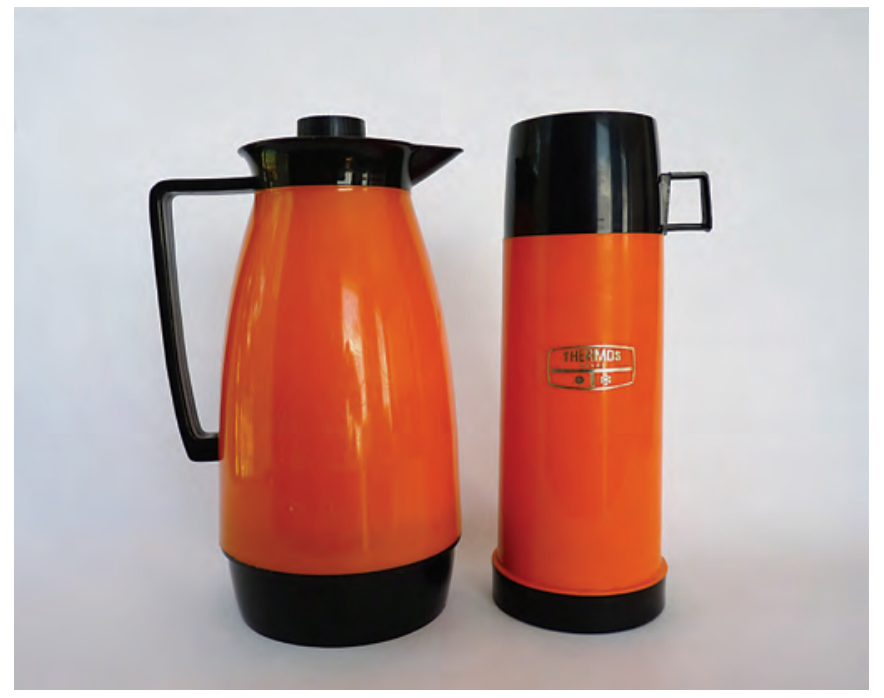

Figure 4. The thermal insulation of a coffee pot.

By the Fourier analysis of the corresponding heat diffusion equation, the decay of the temperature goes as $e^{-t \lambda}$, where $\lambda$ is the first eigenvalue of the elliptic operator written in the weak form as

$\langle\mathcal{A} u, \phi\rangle=\int_{\Omega} \nabla u \nabla \phi d x+\delta \int_{\Sigma_{\varepsilon}} \nabla u \nabla \phi d x \quad \forall \phi \in H_{0}^{1}\left(\Omega \cup \Sigma_{\varepsilon}\right)$.

Therefore, indicating by $\lambda(h)$ the first eigenvalue above, stressing its dependence on the function $h$, Problem 2 reduces to finding

$$
\min \left\{\lambda(h): h \in \mathcal{H}_{m}\right\},
$$

where $\mathcal{H}_{m}$ is the class of admissible choices introduced in (3).

\section{The Asymptotic Problem}

In order to simplify our problems of optimal insulation, following a 1974 paper of E. Sanchez-Palencia, we consider the asymptotic model when both the thickness $\varepsilon$ of the insulating layer as well as the conductivity coefficient $\delta$ of the insulator tend to zero. Following the so-called Gammaconvergence approach of earlier work of G. Buttazzo with E. Acerbi, we consider the functionals

$\frac{1}{2} \int_{\Omega}|\nabla u|^{2} d x+\frac{\delta}{2} \int_{\Sigma_{\varepsilon}}|\nabla u|^{2} d x \quad u \in H_{0}^{1}\left(\Omega \cup \Sigma_{\varepsilon}\right)$.

- When $\varepsilon \ll \delta$ the temperature goes to 0 at the boundary and the limit problem is the Dirichlet one, related to the functional

$$
\frac{1}{2} \int_{\Omega}|\nabla u|^{2} d x \quad u \in H_{0}^{1}(\Omega) .
$$

- When $\varepsilon \gg \delta$ the normal derivative of the temperature goes to 0 at the boundary and the limit problem is the Neumann one, related to the functional

$$
\frac{1}{2} \int_{\Omega}|\nabla u|^{2} d x \quad u \in H^{1}(\Omega) .
$$

- When $\varepsilon \approx k \delta$ with $k>0$ the limit problem is a Robin type problem, involving both Dirichlet and Neumann boundary conditions, related to the functional

$$
\frac{1}{2} \int_{\Omega}|\nabla u|^{2} d x+\frac{1}{2 k} \int_{\partial \Omega} \frac{u^{2}}{h} d \sigma \quad u \in H^{1}(\Omega) .
$$

In the rest of this article we are in the framework of the last situation. We can reformulate Problems 1 and 2 in their asymptotic form, as $\varepsilon$ and $\delta$ go to zero, with $k \approx \varepsilon / \delta$.

The asymptotic form of Problem 1 becomes

$$
\min \left\{\mathscr{\varphi}(h): h \in \mathcal{H}_{m}\right\},
$$

where $\mathcal{H}_{m}$ is given in (3) and $\mathscr{\wp}$ is the asymptotic energy

$$
\begin{array}{r}
\mathscr{\varphi}(h)=\min \left\{\frac{1}{2} \int_{\Omega}|\nabla u|^{2} d x+\frac{1}{2 k} \int_{\partial \Omega} \frac{u^{2}}{h} d \sigma-\int_{\Omega} f u d x:\right. \\
\left.u \in H^{1}(\Omega)\right\} .
\end{array}
$$

The Euler-Lagrange equation of the minimum problem above is

$$
\left\{\begin{array}{l}
-\Delta u=f \quad \text { in } \Omega \\
\frac{1}{k} u+h \frac{\partial u}{\partial v}=0 \quad \text { on } \partial \Omega .
\end{array}\right.
$$

Denoting by $u_{h}$ its solution, multiplying both sides by $u_{h}$, and integrating by parts gives that

$$
\mathscr{\wp}(h)=-\frac{1}{2} \int_{\Omega} f u_{h} d x .
$$
by

Analogously, the asymptotic form of Problem 2 is given

where $\lambda(h)$ is the first eigenvalue of the elliptic operator written in a weak form as

$$
\langle\mathcal{A} u, \phi\rangle=\int_{\Omega} \nabla u \nabla \phi d x+\frac{1}{k} \int_{\partial \Omega} \frac{u \phi}{h} d \sigma \quad \forall \phi \in H^{1}(\Omega) .
$$




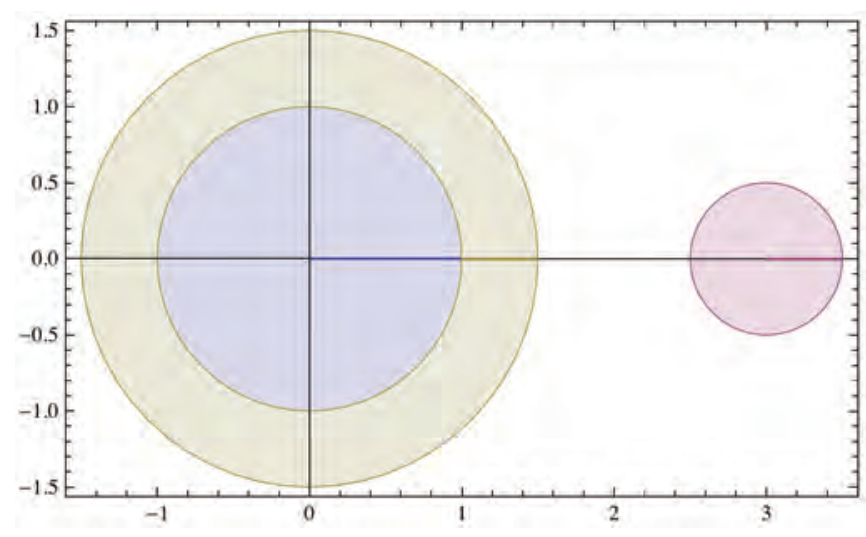

Figure 5. The optimal insulation of a single ball with a uniform heat source has constant thickness as on the left. For two balls of different sizes, place all the insulation on the larger one.

Equivalently, $\lambda(h)$ can be written in terms of the Rayleigh quotient

$$
\begin{aligned}
& \lambda(h)= \\
& \min \left\{\frac{\int_{\Omega}|\nabla u|^{2} d x+\frac{1}{k} \int_{\partial \Omega} \frac{u^{2}}{h} d \sigma}{\int_{\Omega} u^{2} d x}: u \in H^{1}(\Omega), u \neq 0\right\} .
\end{aligned}
$$

\section{Energy Optimization}

The optimization problem (5) is a double minimization problem:

$\min _{h \in \mathcal{H}_{m}} \min _{u \in H^{1}(\Omega)}\left\{\frac{1}{2} \int_{\Omega}|\nabla u|^{2} d x+\frac{1}{2 k} \int_{\partial \Omega} \frac{u^{2}}{h} d \sigma-\int_{\Omega} f u d x\right\}$.

Interchanging the two minima we have that for every $u \in H^{1}(\Omega)$ that does not identically vanish on $\partial \Omega$ the best choice for $h$ is

$$
h=m \frac{|u|}{\int_{\partial \Omega}|u| d \sigma},
$$

while the choice of $h$ is irrelevant when $u \in H_{0}^{1}(\Omega)$. This reduces the minimization problem (5) to

$$
\begin{array}{r}
\min \left\{\frac{1}{2} \int_{\Omega}|\nabla u|^{2} d x+\frac{1}{2 k m}\left(\int_{\partial \Omega}|u| d \sigma\right)^{2}\right. \\
\left.-\int_{\Omega} f u d x: u \in H^{1}(\Omega)\right\} .
\end{array}
$$

Theorem 1 ([2], [1]). Assume $\Omega$ is connected. Then the functional

$$
F(u)=\frac{1}{2} \int_{\Omega}|\nabla u|^{2} d x+\frac{1}{2 k m}\left(\int_{\partial \Omega}|u| d \sigma\right)^{2}
$$

is strictly convex on $H^{1}(\Omega)$. Hence for every $f \in L^{2}(\Omega)$ the minimization problem (7) admits a unique solution $\bar{u}$. Thus the optimal function $h_{m}$ for problem (5) is given by

$$
h=m \frac{|\bar{u}|}{\int_{\partial \Omega}|\bar{u}| d \sigma} .
$$

By uniqueness, if $\Omega=B_{R}$ in $\mathbb{R}^{d}$ and $f=1$ the optimal solution $\bar{u}$ above is radial:

$$
\bar{u}(r)=\frac{R^{2}-r^{2}}{2 d}+\frac{k m}{d^{2} \omega_{d} R^{d-2}},
$$

hence, the optimal thickness $h_{m}$ is constant.

If $\Omega$ is not connected the optimal insulation strategy is different. Let $\Omega=B_{R_{1}} \cup B_{R_{2}}$ in $\mathbb{R}^{d}$ (union of two disjoint balls) and $f=1$. Then:

- if $R_{1}=R_{2}=R$, any choice of $h_{m}$ constant around $B_{R_{1}}$ and on $B_{R_{2}}$ is optimal;

- if $R_{1} \neq R_{2}$, then the optimal choice is to concentrate all the insulator around the largest ball, with constant thickness, leaving the smallest ball unprotected as in Figure 5.

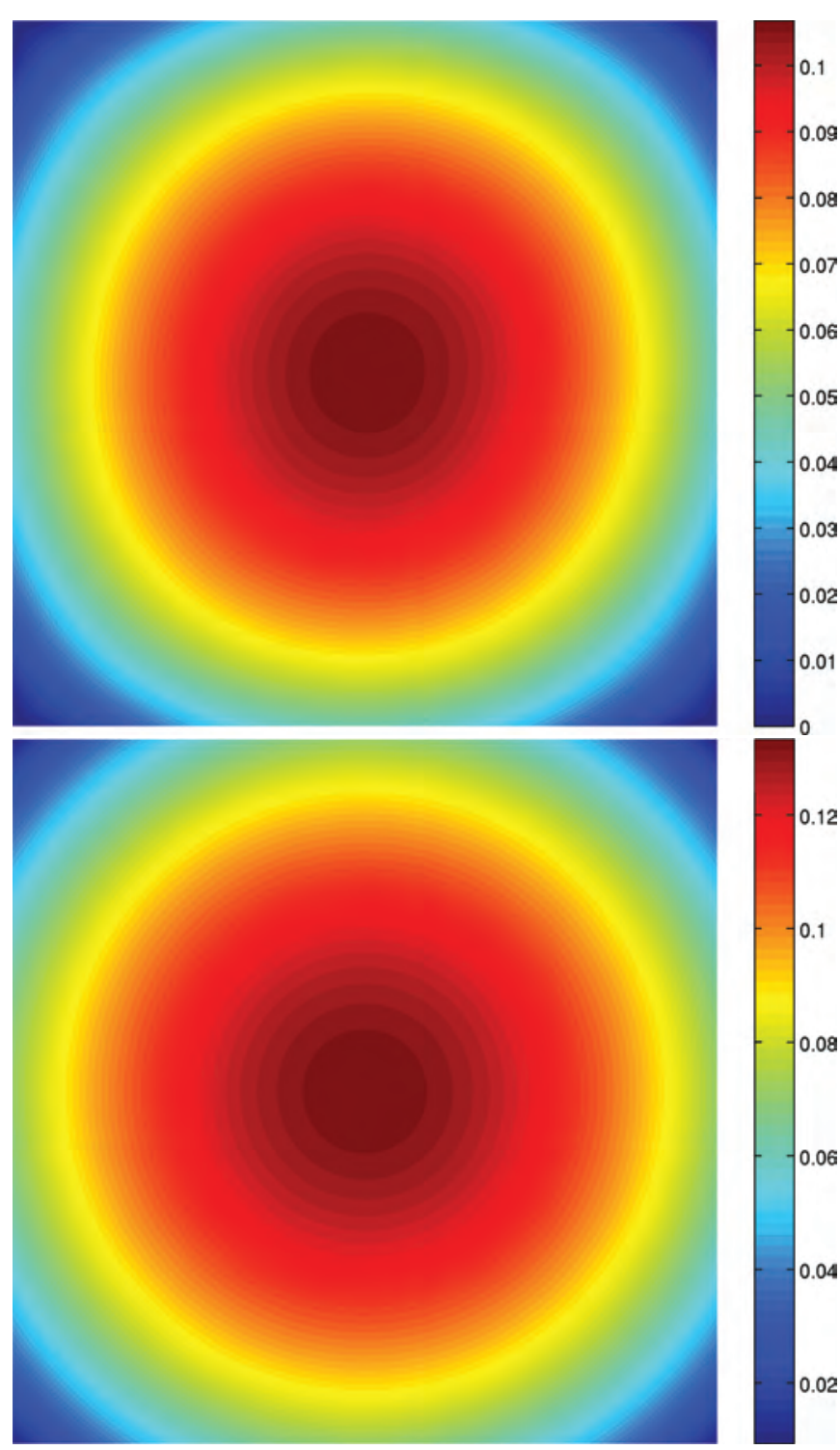

Figure 6. For a square, it is best to concentrate the insulation on the middles of the sides, especially when the total amount of insulation $m$ is small ( $m=1$ on the top and $m=2$ on the bottom). 
For a square with a uniform heat source, numerical computation shows that it is best to concentrate the insulation on the middles of the sides, leaving the corners relatively cool, as in Figure 6.

It is interesting to study the behavior of the thickness $h_{m}$ as the total amount of insulation $m \rightarrow 0$. P. Esposito and G. Riey [4] showed that the rescaled functions $h_{m} / m$ weakly converge as measures to a probability measure concentrated on the set where the normal derivative $\partial u_{0} / \partial v$ reaches its minimal value, where $u_{0}$ is the solution of the Dirichlet problem

$$
-\Delta u=f, \quad u \in H_{0}^{1}(\Omega) .
$$

For instance, when $\Omega$ is a square, the region of concentration of $h_{m} / m$ consists of the four middle points of the sides.

\section{Eigenvalue Optimization}

The second optimization problem (6) looks very similar to the first one, but its solutions behave very differently. A 1999 paper [3] claimed that for a ball the optimal insulation is uniform, but the argument works only if the total quantity $m$ of insulation is large enough [1].

Like problem (5), problem (6) is a double minimization problem; the optimal function $h_{m}$ is given by

$$
h_{m}=m \frac{|\bar{u}|}{\int_{\partial \Omega}|\bar{u}| d \sigma},
$$

where $\bar{u}$ solves the auxiliary variational problem

$$
\begin{array}{r}
\min \left\{\int_{\Omega}|\nabla u|^{2} d x+\frac{1}{k m}\left(\int_{\partial \Omega}|u| d \sigma\right)^{2}: u \in H^{1}(\Omega),\right. \\
\left.\int_{\Omega} u^{2} d x=1\right\} .
\end{array}
$$

Theorem 2 ([1]). For every $\Omega$ there exists a solution $h_{m}$ to the optimization problem (6). If $\Omega=B_{R}$ there exists a threshold $m_{0}>0$ such that:

- if $m>m_{0} \bar{u}$ is radial, then $h_{m}$ is constant;

- if $m<m_{0} \bar{u}$ is not radial, then $h_{\text {opt }}$ is not constant.

The threshold value $m_{0}$ is determined as the unique $m$ such that $\lambda_{m}=\Lambda$, where

$$
\begin{aligned}
\lambda_{m}=\min \left\{\int_{\Omega}|\nabla u|^{2} d x+\right. & \frac{1}{k m}\left(\int_{\partial \Omega}|u| d \sigma\right)^{2}: \\
& \left.u \in H^{1}(\Omega), \int_{\Omega} u^{2} d x=1\right\},
\end{aligned}
$$

while $\Lambda$ is the first nonzero Neumann eigenvalue

$$
\begin{aligned}
\Lambda=\min \left\{\int_{\Omega}|\nabla u|^{2} d x:\right. & u \in H^{1}(\Omega), \\
& \left.\int_{\Omega} u^{2} d x=1, \int_{\Omega} u d x=0\right\} .
\end{aligned}
$$

When the dimension $d=1$ no symmetry breaking occurs. In fact, in this case, the first nonzero Neumann eigenvalue $\Lambda$ coincides with the first Dirichlet eigenvalue $\Lambda_{0}$ and so $\lambda_{m}<\Lambda$ for every amount of insulation $m$.

Figure 7 shows computational optimal distributions for the unit disk and $k=1$, for two values of the total insulation $m$. By (8), the insulation thickness is proportional to the temperature.

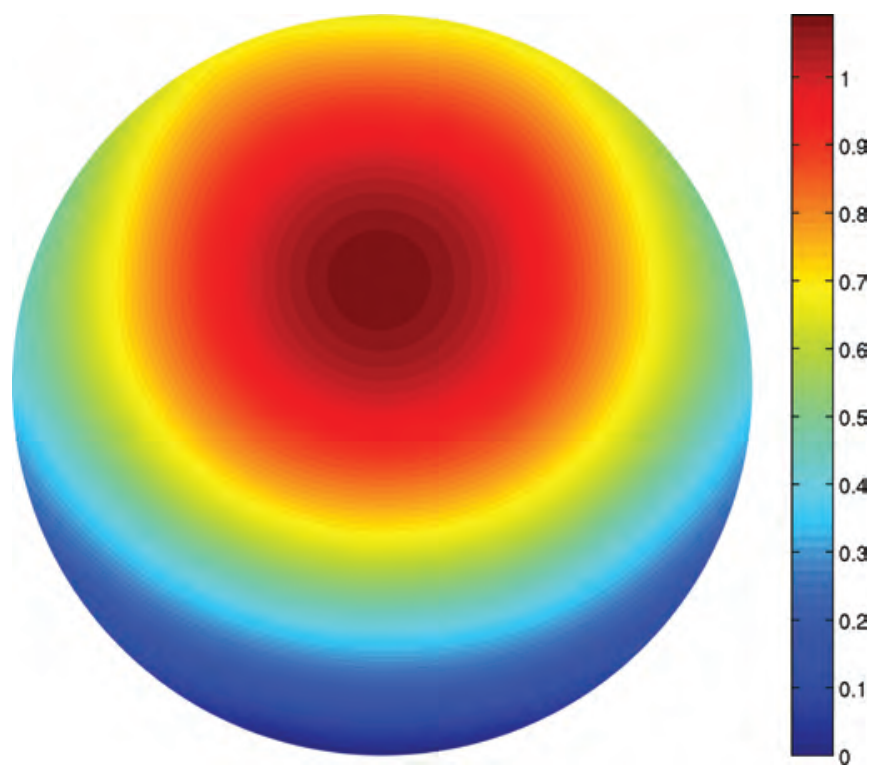

Figure 7. Optimal eigenvalue insulation, proportional to boundary thickness, breaks symmetry when the total insulation is below a certain threshold.

\section{Some Open Problems}

In the presentation above we always considered $\Omega$ fixed; however it would be very interesting to seek the optimal shapes as well as the optimal ways of insulating them. More precisely, denoting by $\mathscr{\ell}(\Omega)$ and by $\lambda(\Omega)$ the minimal values of problems 1 and 2

$$
\begin{array}{r}
\mathscr{\varphi}(\Omega)=\min \left\{\frac{1}{2} \int_{\Omega}|\nabla u|^{2} d x+\frac{1}{2 k m}\left(\int_{\partial \Omega}|u| d \sigma\right)^{2}\right. \\
\left.-\int_{\Omega} f u d x: u \in H^{1}(\Omega)\right\}, \\
\lambda(\Omega)=\min \left\{\int_{\Omega}|\nabla u|^{2} d x+\frac{1}{k m}\left(\int_{\partial \Omega}|u| d \sigma\right)^{2}:\right. \\
\left.u \in H^{1}(\Omega), \int_{\Omega} u^{2} d x=1\right\},
\end{array}
$$

the two shape optimization problems read as

$$
\begin{aligned}
& \min \{\mathscr{\varnothing}(\Omega):|\Omega|=M\}, \\
& \min \{\lambda(\Omega):|\Omega|=M\} .
\end{aligned}
$$

The problems above look very difficult and we do not even have proofs that optimal shapes exist. It would be very interesting to prove (or disprove) the following facts.

- In the case of energy with a general heat source $f$ show that an optimal shape exists.

- In the case of energy with a uniform heat source show that the optimal shape is a ball. We can actually prove that the ball is stationary with respect to smooth perturbations of the boundary.

- In the case of the eigenvalue for temperature decay in dimension $d=2$, show that an optimal shape exists. If $d \geq 3$ it is easy to see that, taking $\Omega$ as the union of many disjoint small balls, the value $\lambda(\Omega)$ can be made arbitrarily close to zero. 
- In the case of the eigenvalue for temperature decay in dimension $d=2$, the optimal shape is a ball if $m$ is large $\left(m>m_{0}\right)$.

- In the case of the eigenvalue for temperature decay in dimension $d=2$, characterize the optimal shape (if any) when $m$ is small $\left(m<m_{0}\right)$. We can prove that if $m<m_{0}$ the ball cannot be optimal or even a stationary domain with respect to smooth perturbations of the boundary.

\section{Acknowledgement}

We wish to thank Beniamin Bogosel for the help provided in the numerical simulations.

\section{References}

[1] D. Bucur, G. ButTazzo, and C. Nitsch, Symmetry breaking for a problem in optimal insulation, J. Math. Pures Appl. (9) 107 (2017), no. 4, 451-463. MR 3623640

[2] G. ButTAzzo, Thin insulating layers: the optimization point of view, Proceedings of "Material Instabilities in Continuum Mechanics and Related Mathematical Problems", Edinburgh 1985-1986, edited by J. M. Ball, Oxford University Press, Oxford (1988), 11-19. MR 0970514

[3] S.J. Cox, B. KAwoHL, and P. X. Uhlig, On the optimal insulation of conductors, J. Optim. Theory Appl. 100 (1999), no. 2, 253-263. MR 1673432

[4] P. Esposito and G. RiEY, Asymptotic behaviour of a thin insulation problem, J. Convex Anal. 10 (2003), 379-388. MR 2043863

[5] A. FRIEDMAN, Reinforcement of the principal eigenvalue of an elliptic operator, Arch. Rational Mech. Anal. 73 (1980), no. 1, 1-17. MR 555579

\section{Photo Credits}

Figure 1 cLutz Weidner.

Figure 2 (C) Sönke Kraft, aka Arnulf zu Linden (Own work).

Figure 3 (c) David McKelvey.

Figure 4 (c) Gail Thomas.

Figures 1-4 used according to the terms of the Creative Commons License.

Figures 5-7 and all headshots courtesy of the authors.

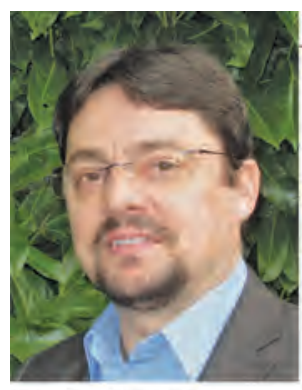

Dorin Bucur

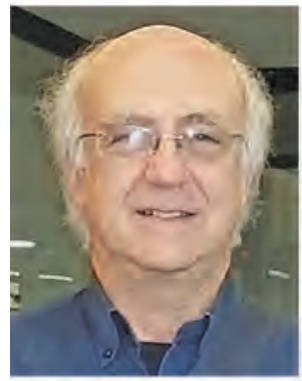

Giuseppe

Buttazzo

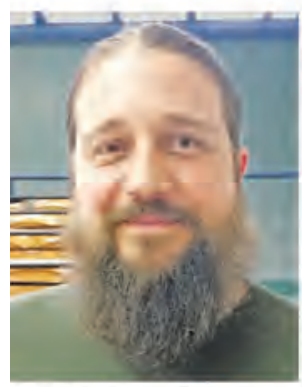

Carlo Nitsch

\section{ABOUT THE AUTHORS}

After graduate studies in Timisoara and Nice, Dorin Bucur got his $\mathrm{PhD}$ at École des Mines de Paris in 1995 and became CNRS researcher at Besançon. Currently he is professor at Université Savoie Mont Blanc and senior member of Institut Universitaire de France. His main research interests are free boundary problems and PDEs.

Giuseppe Buttazzo earned his $\mathrm{PhD}$ at Scuola Normale Superiore di Pisa in 1980 under Ennio De Giorgi. His main research interests are calculus of variations, PDEs, and optimization. In 2011 he received the Luigi and Wanda Amerio prize.

Carlo Nitsch received his $\mathrm{PhD}$ at University of Rome in 2004 under Michiel Bertsch and Grigory Isaakovich Barenblatt. Winner of the Carlo Miranda Prize (2008) and a Humboldt Fellowship (2013), he has written more than forty papers on elliptic eigenvalue problems, functional inequalities, and PDEs. 